\title{
On the Convergence of a Time Discretization Scheme for the Navier-Stokes Equations*
}

\author{
By T. Geveci
}

\begin{abstract}
A linearized version of the implicit Euler scheme is considered for the approximation of the solutions to the Navier-Stokes equations in a two-dimensional domain. The rate of convergence in the $H^{1}$-norm is established.
\end{abstract}

1. Introduction. We are concerned with the discretization in time of the Navier-Stokes equations in a bounded two-dimensional domain:

$$
\begin{aligned}
& \frac{\partial u}{\partial t}(t, x)-\Delta u(t, x)+\nabla p(t, x)+(u \cdot \nabla) u(t, x)=0, \quad x \in \Omega, t>0, \\
& \operatorname{div} u(t, x)=0, \quad x \in \Omega, t>0, \\
& u(t, x)=0, \quad x \in \partial \Omega, t>0, \\
& u(0, x)=u_{0}(x), \quad x \in \Omega .
\end{aligned}
$$

Here, $u(t, x)=\left(u_{1}(t, x), u_{2}(t, x)\right)$ is the velocity, $p(t, x)$ is the pressure, $\Omega$ is a bounded domain in $\mathbf{R}^{2}$ with smooth boundary $\partial \Omega$, and $u_{0}$ is the initial velocity field.

As in Fujita and Kato [5], [15] and Temam [24], we cast (1.1) as an evolution equation in the appropriate Hilbert space:

$\mathscr{V}=\left\{v=\left(v_{1}, v_{2}\right): v_{1}, v_{2} \in C_{0}^{\infty}(\Omega), \operatorname{div} v=0\right\}$

$H=$ closure of $\mathscr{V}$ in $L^{2,2}(\Omega)$, the space of $\mathbf{R}^{2}$-valued functions, each component of which is in $L^{2}(\Omega)$, equipped with the inner product

$$
(u, v)=\int_{\Omega} \sum_{i=1}^{2} u_{i}(x) v_{i}(x) d x
$$

and the induced norm $\|u\|=(u, u)^{1 / 2}$;

$V=$ closure of $\mathscr{V}$ in $H_{0}^{1 / 2}(\Omega)$, the Sobolev space of $\mathbf{R}^{2}$-valued functions, each component of which is in $H_{0}^{1}(\Omega)$, equipped with the inner product

$$
(u, v)_{1}=\int_{\Omega} \sum_{i, j=1}^{2} \frac{\partial u_{i}}{\partial x_{j}} \frac{\partial v_{i}}{\partial x_{j}} d x
$$

and the induced norm $\|u\|_{1}=(u, u)_{1}^{1 / 2}$.

Similarly, the spaces $H^{s, 2}(\Omega)$ and the norms $\|\cdot\|_{s}$ are defined in terms of the standard Sobolev spaces.

Received September 25, 1987; revised April 22, 1988.

1980 Mathematics Subject Classification (1985 Revision). Primary 35Q10, 65M10.

${ }^{*}$ Research supported in part by NSF Grant DMS-8701109. 
We let $P: L^{2,2}(\Omega) \rightarrow H$ denote the orthogonal projection and define the Stokes operator $A: D(A) \subset H \rightarrow H, D(A)=V \cap H^{2,2}(\Omega)$ by $A u=-P \Delta u, u \in D(A)$. We note that $\|u\|_{s}$ and $\left\|A^{s / 2} u\right\|$ are equivalent, $u \in D\left(A^{s / 2}\right), 0 \leq s \leq 2$ [7].

Within this framework, (1.1) is expressed as the evolution equation in $H$ : $u(t) \in D(A), t \geq 0$, and

$$
\frac{d u}{d t}(t)+A u(t)+B(u(t), u(t))=0, \quad t>0, \quad u(0)=u_{0},
$$

where $B(u, v)=P(u \cdot \nabla) v$.

The application of a linearized version of the implicit Euler scheme to (1.2) determines the sequence $u_{k, n} \in D(A), n=0,1,2, \ldots$, such that

$$
\bar{\partial}_{t} u_{k, n}+A u_{k, n}+B\left(u_{k, n-1}, u_{k, n}\right)=0, \quad n=1,2, \ldots, \quad u_{k, 0}=u_{0},
$$

where $k>0$ is the time step and

$$
\bar{\partial}_{t} u_{k, n}=\frac{u_{k, n}-u_{k, n-1}}{k} .
$$

We will establish the following result:

THEOREM. If $u_{0} \in D(A)$ and $t=n k$, then

$$
\left\|u_{k, n}-u(t)\right\|_{1} \leq \frac{C e^{-\delta t}}{t^{1 / 2}} k
$$

for $k<k_{0}$, where $C, \delta$ and $k_{0}$ are positive constants depending on the data $u_{0}$ and $\Omega$ only.

Here and in the sequel, $C, \delta$ and $k_{0}$ will denote possibly different constants which depend only on the data. This convention renders the proofs of results such as the above theorems more readable. In any case, the interested reader should have no difficulty in tracing the dependence of the various constants on the data.

The above result parallels results pertaining to the approximation by the implicit Euler scheme of the analytic semigroup generated by the positive definite selfadjoint operator $A$, as discussed, for example, by Fujita and Mizutani [6] and Thomée [25].

The convergence in the $L^{2}$-norm of the scheme described by (1.3) has been discussed by Girault and Raviart [8]. They have established the $L^{2}$-convergence of the scheme in terms of the smoothness properties of the solution. Under the same conditions as in the theorem, and using the same techniques, we are able to show that

$$
\left\|u_{k, n}-u(t)\right\| \leq C e^{-\delta t} k, \quad t=n k,
$$

for $k<k_{0}$, where $C, \delta$ and $k_{0}$ are positive constants depending on the data $u_{0}$ and $\Omega$ only. We do not include the proof since it is straightforward, once (1.4) is established, and (1.5) is not sufficiently novel.

Earlier, Temam [22] derived a priori bounds for the scheme (1.3) and concluded qualitative convergence without a convergence rate in weak norms. Rannacher [19] gave $O(\Delta t)$-error estimates for the explicit Euler scheme. Recently, Heywood and Rannacher [12] obtained local and global $O\left(\Delta t^{2}\right)$-error estimates for the CrankNicolson scheme under realistic assumptions concerning the smoothness of the solution. It is relatively easier to establish the rate of convergence of higher-order schemes by assuming the solution to be sufficiently regular. However, as has been 
emphasized by Heywood and Rannacher in a series of papers [9], [10], [11], [12], and discussed also by Rautmann [21] and Temam [23], [24], such regularity assumptions may entail global compatibility conditions which are not met or which are not verifiable, in general. Higher-order results as in [2], [8] and [16] involve such conditions, and future work should attempt to clarify whether the anticipated orders of such schemes are realized under realistic assumptions on the data.

Our approach is based on the Fujita-Kato approach to the Navier-Stokes equations [5], [15], and has been inspired by Okamoto's papers [17], [18] on the spatial discretization of (1.2). We have not considered fully discrete schemes since the technicalities, which are considerable, vary depending on the spatial discretization schemes that are utilized, and may obscure the essential goal of the paper, i.e., the demonstration of the convergence of the linearized implicit Euler scheme (1.3) at the predicted rate for $u_{0} \in D(A)$. By the same token, we have not included nonhomogeneous boundary data or a forcing term in (1.1). Under appropriate technical assumptions, the basic result (1.4) may be extended to the nonhomogeneous cases. If $\Omega \subset \mathbf{R}^{3}$, the counterpart of our theorem may be established over a time interval $(0, T]$ in which a bound on $\|u(t)\|_{1}$ and $\left\|u_{n, k}\right\|_{1}(t=n k)$ may be assumed. In the 2 -dimensional case, the required a priori estimates are available for $\|u(t)\|_{1}$ and will be established in the next section for $\left\|u_{n, k}\right\|_{1}$.

\section{Some a priori Estimates.}

LEMMA 1. If $\left\{u_{k, n}\right\}_{n=0}^{\infty}$ is the solution of the linearized implicit Euler scheme (1.3), the following a priori estimates are valid:

$$
\begin{gathered}
\left\|u_{k, n}\right\|^{2}+2 \sum_{j=1}^{n}\left\|A^{1 / 2} u_{k, j}\right\|^{2} k \leq\left\|u_{0}\right\|^{2}, \quad n=1,2, \ldots \\
\left\|A^{1 / 2} u_{k, n}\right\| \leq C\left(\left\|A^{1 / 2} u_{0}\right\|, \Omega\right) e^{-\delta t}, \quad 0<k<k_{0}
\end{gathered}
$$

where $t=n k, C, \delta$ and $k_{0}$ are positive constants which depend on the data $u_{0}$ and $\Omega$.

Proof. We form the inner product of (1.3) with $u_{k, n}$ and obtain

$$
\left(\bar{\partial}_{t} u_{k, n}, u_{k, n}\right)+\left(A u_{k, n}, u_{k, n}\right)+\left(B\left(u_{k, n-1}, u_{k, n}\right), u_{k, n}\right)=0 .
$$

Since

$$
\left(B\left(u_{k, n-1}, u_{k, n}\right), u_{k, n}\right)=b\left(u_{k, n-1}, u_{k, n}, u_{k, n}\right)=0
$$

as in Temam [22, p. 163], and

$$
\left(\bar{\partial}_{t} u_{k, n}, u_{k, n}\right)=\frac{1}{2} \bar{\partial}_{t}\left\|u_{k, n}\right\|^{2}+\frac{k}{2}\left\|\bar{\partial}_{t} u_{k, n}\right\|^{2}
$$

as in Thomée [25, p. 157], (2.3) yields

$$
\frac{1}{2} \bar{\partial}_{t}\left\|u_{k, n}\right\|^{2}+\left\|A^{1 / 2} u_{k, n}\right\|^{2} \leq 0,
$$


so that

$$
\left\|u_{k, n}\right\|^{2}+2 \sum_{j=1}^{n}\left\|A^{1 / 2} u_{k, j}\right\|^{2} k \leq\left\|u_{0}\right\|^{2},
$$

i.e., (2.1) is established.

In order to establish the a priori bound (2.2) on $\left\|A^{1 / 2} u_{k, n}\right\|$, we form the inner product of (1.3) with $A u_{k, n}$ and obtain

$$
\left(\bar{\partial}_{t} u_{k, n}, A u_{k, n}\right)+\left\|A u_{k, n}\right\|^{2}+\left(B\left(u_{k, n-1}, u_{k, n}\right), A u_{k, n}\right)=0,
$$

so that

$$
\begin{aligned}
& \frac{1}{2} \bar{\partial}_{t}\left\|A^{1 / 2} u_{k, n}\right\|^{2}+\left\|A u_{k, n}\right\|^{2} \leq\left\|B\left(u_{k, n-1}, u_{k, n}\right)\right\|\left\|A u_{k, n}\right\| \\
& \quad \leq C\left\|u_{k, n-1}\right\|^{1 / 2}\left\|A^{1 / 2} u_{k, n-1}\right\|^{1 / 2}\left\|A^{1 / 2} u_{k, n}\right\|^{1 / 2}\left\|A u_{k, n}\right\|^{3 / 2}
\end{aligned}
$$

where we have used the inequality

$$
\|v\|_{L^{4}} \leq C\|v\|^{1 / 2}\|v\|_{1}^{1 / 2}, \quad v \in H^{1}(\Omega), \Omega \subset \mathbf{R}^{2}
$$

[22, p. 291].

Making use of Young's inequality, (2.7) leads to

$$
\begin{aligned}
\left\|A^{1 / 2} u_{k, n}\right\|^{2}+k\left\|A u_{k, n}\right\|^{2} \leq & \left\|A^{1 / 2} u_{k, n-1}\right\|^{2} \\
& +C k\left\|u_{k, n-1}\right\|^{2}\left\|A^{1 / 2} u_{k, n-1}\right\|^{2}\left\|A^{1 / 2} u_{k, n}\right\|^{2},
\end{aligned}
$$

and summing over $n$, using the inequality $\left\|A^{1 / 2} u_{k, n}\right\| \leq C\left\|A u_{k, n}\right\|$, we obtain

$$
\begin{aligned}
\left\|A^{1 / 2} u_{k, n}\right\|^{2} \leq & \left\|A^{1 / 2} u_{0}\right\|^{2} \\
& +C k \sum_{m=1}^{n}\left[\left\|u_{k, m-1}\right\|^{2}\left\|A^{1 / 2} u_{k, m-1}\right\|^{2}-1\right]\left\|A^{1 / 2} u_{k, m}\right\|^{2} .
\end{aligned}
$$

The inequalities (2.1), (2.8) and the discrete Gronwall lemma, as for example in [13], lead to the a priori estimate (2.2).

In addition to the estimates on the solution of (1.2) that we will be able to refer to, we will need the following estimate:

LEMMA 2. If $u_{0} \in D(A)$, then

$$
\left\|A^{1 / 2} D_{t} u(t)\right\| \leq \frac{C\left(\left\|A u_{0}\right\|, \Omega\right)}{t^{1 / 2}} e^{-\delta t}, \quad t>0 .
$$

We omit the proof since it is readily obtainable using the techniques of [5] and $[18]$.

3. The Error Estimate. In this section we will prove the theorem in Section 1. We will freely use the results of Fujita and Kato [5], [15], Fujita and Morimoto [7], Temam [22], [24], and Foias and Temam [4] with regard to the fractional powers of the Stokes operator $A$ and the properties of the trilinear form $b(u, v, w)=(B(u, v), w)$. The a priori estimates that have been established by Okamoto [18] for the solution of (1.2) are essential as well. The reader will notice the parallels between our treatment of time discretization and Okamoto's treatment of spatial discretization. 
We restate the theorem:

THEOREM. If $u_{0} \in D(A)$ and $t=n k, n=1,2, \ldots$, then

$$
\left\|u_{k, n}-u(t)\right\|_{1} \leq \frac{C\left(\left\|A u_{0}\right\|, \Omega\right) e^{-\delta t}}{t^{1 / 2}} k .
$$

Proof. We have

$$
\begin{aligned}
& u(t)=e^{-t A} u_{0}-\int_{0}^{t} e^{-(t-s) A} B(u(s), u(s)) d s, \quad t=n k \\
& u_{k, n}=E_{k}^{n} u_{0}-\sum_{j=1}^{n} E_{k}^{n-j+1} B\left(u_{k, j-1}, u_{k, j}\right) k
\end{aligned}
$$

where $E_{k}=(I+k A)^{-1}, I$ denoting the identity.

As in Thomée [25],

$$
\left\|A^{1 / 2}\left(E_{k}^{n}-e^{-t A}\right) u_{0}\right\| \leq \frac{C\left(\left\|A u_{0}\right\|, \Omega\right) e^{-\delta t}}{t^{1 / 2}} k
$$

where, as always, $\delta>0$ also depends on the data. Thus,

$$
\begin{aligned}
\left\|A^{1 / 2}\left(u(t)-u_{k, n}\right)\right\| \leq \frac{C e^{-\delta t}}{t^{1 / 2}} k+\| & A^{1 / 2} \int_{0}^{t} e^{-(t-s) A} B(u(s), u(s)) d s \\
& -A^{1 / 2} \sum_{j=1}^{n} E_{k}^{n-j+1} B\left(u_{k, j-1}, u_{k, j}\right) k \| .
\end{aligned}
$$

We write

$$
\begin{aligned}
\int_{0}^{t} e^{-(t-s) A} B(u(s), u(s)) d s-\sum_{j=1}^{n} E_{k}^{n-j+1} B\left(u_{k, j-1}, u_{k, j}\right) k \\
=\left[\int_{0}^{t} e^{-(t-s) A} B(u(s), u(s)) d s-\sum_{j=1}^{n} E_{k}^{n-j+1} B\left(u_{j-1}, u_{j}\right) k\right] \\
+\left[\sum_{j=1}^{n} E_{k}^{n-j+1}\left(B\left(u_{j-1}, u_{j}\right)-B\left(u_{k, j-1}, u_{k, j}\right)\right) k\right]
\end{aligned}
$$

where $u_{j}$ denotes $u(j k)$.

We write the last expression of (3.6) as

$$
\begin{aligned}
\sum_{j=1}^{n} E_{k}^{n-j+1}\left(B\left(u_{j-1}, u_{j}\right)-B\left(u_{k, j-1}, u_{k, j}\right)\right) k \\
=\sum_{j=1}^{n} E_{k}^{n-j+1} B\left(u_{j-1}-u_{k, j-1}, u_{j}\right) k+\sum_{j=1}^{n} E_{k}^{n-j+1} B\left(u_{k, j-1}, u_{j}-u_{k, j}\right) k \\
=\sum_{j=1}^{n-1} E_{k}^{n-j} B\left(u_{j}-u_{k, j}, u_{j+1}\right) k+\sum_{j=1}^{n-1} E_{k}^{n-j+1} B\left(u_{k, j-1}, u_{j}-u_{k, j}\right) k \\
\quad+E_{k} B\left(u_{k, n-1}, u_{n}-u_{k, n}\right) k
\end{aligned}
$$


since $u_{k, 0}=u_{0}$. We have

$$
\begin{aligned}
& \left\|A^{1 / 2} E_{k} B\left(u_{k, n-1}, u_{n}-u_{k, n}\right)\right\| k \\
& \quad=\left\|A^{3 / 4} E_{k} A^{-1 / 4} B\left(u_{k, n-1}, u_{n}-u_{k, n}\right)\right\| k \\
& \quad \leq \frac{C}{k^{3 / 4}}\left\|A^{1 / 4} u_{k, n-1}\right\| \cdot\left\|A^{1 / 2}\left(u_{n}-u_{k, n}\right)\right\| k \leq C k^{1 / 4}\left\|A^{1 / 2}\left(u_{n}-u_{k, n}\right)\right\|
\end{aligned}
$$

thanks to the estimate $(2.2)$ on $\left\|A^{1 / 2} u_{k, n}\right\|$.

We consider next

$$
\begin{aligned}
& \sum_{j=1}^{n-1}\left\|A^{1 / 2} E_{k}^{n-j} B\left(u_{j}-u_{k, j}, u_{j+1}\right)\right\| k \\
& \quad=\sum_{j=1}^{n-1}\left\|A^{3 / 4} E_{k}^{n-j} A^{-1 / 4} B\left(u_{j}-u_{k, j}, u_{j+1}\right)\right\| k \\
& \leq C e^{-\delta t} \sum_{j=1}^{n-1} \frac{e^{j k \delta}}{(n k-j k)^{3 / 4}}\left\|A^{1 / 4}\left(u_{j}-u_{k, j}\right)\right\|\left\|A^{1 / 2} u_{j+1}\right\| k \\
& \leq C e^{-\delta t} \sum_{j=1}^{n-1} \frac{e^{j k \delta}}{(n k-j k)^{3 / 4}}\left\|A^{1 / 2}\left(u_{j}-u_{k, j}\right)\right\| k,
\end{aligned}
$$

by virtue of the estimate $(2.2)$ on $\left\|A^{1 / 2} u_{n}\right\|$.

Similarly,

$$
\begin{aligned}
& \sum_{j=1}^{n-1}\left\|A^{1 / 2} E_{k}^{n-j+1} B\left(u_{k, j-1}, u_{j}-u_{k, j}\right)\right\| k \\
& \leq C e^{-\delta t} \sum_{j=1}^{n-1} \frac{e^{j k \delta}}{(n k-j k)^{3 / 4}}\left\|A^{1 / 2}\left(u_{j}-u_{k, j}\right)\right\| k .
\end{aligned}
$$

From (3.5), (3.6), (3.7), (3.8), (3.9) and (3.10) we obtain, for sufficiently small $k$,

$$
\begin{aligned}
e^{\delta t}\left\|A^{1 / 2}\left(u_{n}-u_{k, n}\right)\right\| \leq & \frac{C k}{t^{1 / 2}}+C \sum_{j=1}^{n-1} \frac{e^{\delta j k}}{(t-j k)^{3 / 4}}\left\|A^{1 / 2}\left(u_{j}-u_{k, j}\right)\right\| k \\
& +e^{\delta t} \| A^{1 / 2} \int_{0}^{t} e^{-(t-s) A} B(u(s), u(s)) d s \\
& -A^{1 / 2} \sum_{j=1}^{n} E_{k}^{n-j+1} B\left(u_{j-1}, u_{j}\right) k \| .
\end{aligned}
$$

Thanks to a generalization of Gronwall's lemma as in Okamoto [18] and Amann [1], the theorem will be established once we show that

$$
\begin{array}{r}
\left\|A^{1 / 2} \int_{0}^{t} e^{-(t-s) A} B(u(s), u(s)) d s-A^{1 / 2} \sum_{j=1}^{n} E_{k}^{n-j+1} B\left(u_{j-1}, u_{j}\right) k\right\| \\
\leq \frac{C e^{-\delta t}}{t^{1 / 2}} k .
\end{array}
$$


We write

$$
\begin{gathered}
\int_{0}^{t} e^{-(t-s) A} B(u(s), u(s)) d s-\sum_{j=1}^{n} E_{k}^{n-j+1} B\left(u_{j-1}, u_{j}\right) k \\
=\int_{0}^{t} e^{-(t-s) A}(B(u(s), u(s))-B(u(t), u(t))) d s \\
-\sum_{j=1}^{n} E_{k}^{n-j+1}\left(B\left(u_{j-1}, u_{j}\right)-B(u(t), u(t))\right) k \\
+\left[\int_{0}^{t} e^{-(t-s) A}-\sum_{j=1}^{n} E_{k}^{n-j+1} k\right] B(u(t), u(t)) .
\end{gathered}
$$

We will deal with the last line of (3.13) first. As in Kato [14, p. 489],

$$
\int_{0}^{t} e^{-(t-s) A} d s=\left(I-e^{-t A}\right) A^{-1}
$$

It is also easily verified that

$$
\sum_{j=1}^{n} E_{k}^{n-j+1} k=\left(I-E_{k}^{n}\right) A^{-1}
$$

By (3.14) and (3.15),

$$
\begin{gathered}
\left\|A^{1 / 2}\left[\int_{0}^{t} e^{-(t-s) A}-\sum_{j=1}^{n} E_{k}^{n-j+1} k\right] B(u(t), u(t))\right\| \\
=\left\|A^{1 / 2}\left(E_{k}^{n}-e^{-t A}\right) A^{-1} B(u(t), u(t))\right\| \leq \frac{C e^{-\delta t}}{t^{1 / 2}}\|B(u(t), u(t))\| k \\
\leq \frac{C e^{-\delta t}}{t^{1 / 2}}\|A u(t)\|\left\|A^{1 / 2} u(t)\right\| k \leq \frac{C\left(\left\|A u_{0}\right\|, \Omega\right)}{t^{1 / 2}} e^{-\delta t},
\end{gathered}
$$

thanks to the error estimates on the approximation of $\exp (-t A)$ as in Thomée [25] and the a priori estimates established by Okamoto [18].

By (3.13) and (3.16), the inequality (3.12) will be established once we estimate

$$
\begin{aligned}
\| A^{1 / 2} \int_{0}^{t} e^{-(t-s) A}(B(u(s), u(s))-B(u(t), u(t))) d s \\
\quad-\sum_{j=1}^{n} E_{k}^{n-j+1}\left(B\left(u_{j-1}, u_{j}\right)-B(u(t), u(t))\right) k \| .
\end{aligned}
$$


To this end, we write

$$
\begin{aligned}
\int_{0}^{t} e^{-(t-s) A}(B(u(s), u(s))-B(u(t), u(t))) d s \\
\quad-\sum_{j=1}^{n} E_{k}^{n-j+1}\left(B\left(u_{j-1}, u_{j}\right)-B(u(t), u(t))\right) k \\
=\sum_{j=1}^{n} \int_{(j-1) k}^{j k}\left[e^{-(t-s) A}-e^{-(t-(j-1) k) A}\right](B(u(s), u(s))-B(u(t), u(t))) d s \\
\quad+\sum_{j=1}^{n} \int_{(j-1) k}^{j k} e^{-(t-(j-1) k) A}\left(B(u(s), u(s))-B\left(u_{j-1}, u_{j}\right)\right) d s \\
\quad+\sum_{j=1}^{n}\left[e^{-(t-(j-1) k) A}-E_{k}^{n-(j-1)}\right]\left(B\left(u_{j-1}, u_{j}\right)-B(u(t), u(t))\right) k \\
=
\end{aligned}
$$

We will establish that

$$
\left\|A^{1 / 2} I_{l}\right\| \leq \frac{C e^{-\delta t}}{t^{1 / 2}} k, \quad l=1,2,3
$$

and this will conclude the proof.

In order to estimate $\left\|A^{1 / 2} I_{1}\right\|$ we first note that

$$
\begin{aligned}
e^{-(t-s) A}-e^{-(t-(j-1) k) A} & =e^{-(t-s) A}-e^{-(t-s) A} e^{-(s-(j-1) k) A} \\
& =e^{-(t-s) A}\left[I-e^{-(s-(j-1) k) A}\right]
\end{aligned}
$$

for $s \in[(j-1) k, j k)$. Therefore,

$$
\begin{aligned}
& \left\|A^{1 / 2}\left[e^{-(t-s) A}-e^{-(t-(j-1) k) A}\right] G\right\| \\
& \quad=\left\|A^{3 / 2} e^{-(t-s) A} A^{-1}\left(I-e^{-(s-(j-1) k) A}\right) G\right\| \\
& \quad \leq \frac{C e^{-\delta(t-s)}}{(t-s)^{3 / 2}}\|G\| k, \quad s \in[(j-1) k, j k),
\end{aligned}
$$

as in Crouzeix and Thomée [3, proof of Theorem 1].

Making use of Lemma 2 and [18],

$$
\begin{aligned}
\|B(u(s), u(s))-B(u(t), u(t))\| & \leq C\|A u(t)\|\left\|A^{1 / 2}(u(s)-u(t))\right\| \\
& \leq C\left(\left\|A u_{0}\right\|, \Omega\right) e^{-\delta s} \frac{(t-s)}{s^{1 / 2}}
\end{aligned}
$$

Equations (3.17), (3.19) and (3.20) yield

$$
\begin{aligned}
\left\|A^{1 / 2} I_{1}\right\| & \leq C e^{-\delta t} k \sum_{j=1}^{n} \int_{(j-1) k}^{j k} \frac{1}{(t-s)^{1 / 2} s^{1 / 2}} d s \\
& =C e^{-\delta t} \int_{0}^{t} \frac{1}{(t-s)^{1 / 2} s^{1 / 2}} d s \leq C e^{-\delta t} k .
\end{aligned}
$$


In order to estimate $\left\|A^{1 / 2} I_{2}\right\|$, we write

$$
\begin{aligned}
I_{2}= & \int_{0}^{k} e^{-t A} B(u(s), u(s)) d s-e^{-t A} B\left(u_{0}, u_{1}\right) k \\
& +\sum_{j=2}^{n} \int_{(j-1) k}^{j k} e^{-(t-(j-1) k) A} B\left(u(s)-u_{j-1}, u(s)\right) d s \\
& +\sum_{j=2}^{n} \int_{(j-1) k}^{j k} e^{-(t-(j-1) k) A} B\left(u_{j-1}, u(s)-u_{j}\right) d s .
\end{aligned}
$$

We first observe

$$
\left\|A^{1 / 2} e^{-t A} B(u(s), u(s))\right\| \leq \frac{C e^{-\delta t}}{t^{1 / 2}}\left\|A^{1 / 2} u(s)\right\|\|A u(s)\| \leq \frac{C\left(\left\|A u_{0}\right\|, \Omega\right) e^{-\delta t}}{t^{1 / 2}}
$$

so that

$$
\left\|A^{1 / 2} \int_{0}^{k} e^{-t A} B(u(s), u(s)) d s\right\| \leq \frac{C e^{-\delta t}}{t^{1 / 2}} k
$$

Similarly,

$$
\left\|A^{1 / 2} B\left(u_{0}, u_{1}\right)\right\| k \leq \frac{C e^{-\delta t}}{t^{1 / 2}} k .
$$

By (3.22), (3.23) and (3.24) the result (3.18) for $l=1$ will have been established if such an estimate is proven for the remaining terms of (3.22). It will suffice treating

$$
\left\|A^{1 / 2} \sum_{j=2}^{n} \int_{(j-1) k}^{j k} e^{-(t-(j-1) k) A} B\left(u(s)-u_{j-1}, u(s)\right) d s\right\|
$$

since the last term is treated in a similar manner.

For $j=2,3, \ldots, n, s \in[(j-1) k, j k)$,

$$
\begin{aligned}
& \left\|A^{1 / 2} e^{-(t-(j-1) k)} B\left(u(s)-u_{j-1}, u(s)\right)\right\| \\
& \quad \leq \frac{C e^{-\delta t} e^{\delta(j-1) k}}{(t-(j-1) k)^{1 / 2}}\|A u(s)\|\left\|A^{1 / 2}\left(u(s)-u_{j-1}\right)\right\| \\
& \quad \leq \frac{C e^{-\delta t} k}{(t-(j-1) k)^{1 / 2}((j-1) k)^{1 / 2}},
\end{aligned}
$$

again by [18] and Lemma 2 .

By (3.25) we have

$$
\begin{gathered}
\left\|A^{1 / 2} \sum_{j=2}^{n} \int_{(j-1) k}^{j k} e^{-(t-(j-1) k) A} B\left(u(s)-u_{j-1}, u(s)\right) d s\right\| \\
\leq C e^{-\delta t} k\left(\sum_{j=2}^{n} \frac{k}{(t-(j-1) k)^{1 / 2}((j-1) k)^{1 / 2}}\right) \\
\leq C e^{-\delta t} k \int_{0}^{t} \frac{1}{(t-s)^{1 / 2} s^{1 / 2}} d s \leq C e^{-\delta t} k .
\end{gathered}
$$


The last line of (3.22) is treated similarly, and we are left with the task of estimating $\left\|A^{1 / 2} I_{3}\right\|$. We write

$$
\begin{aligned}
I_{3}= & {\left[e^{-t A}-E_{k}^{n}\right]\left(B\left(u_{0}, u_{1}\right)-B(u(t), u(t))\right) k } \\
& +\sum_{j=2}^{n}\left[e^{-(t-(j-1) k) A}-E_{k}^{n-(j-1)}\right] B\left(u_{j-1}-u(t), u_{j}\right) k \\
& +\sum_{j=2}^{n}\left[e^{-(t-(j-1) k) A}-E_{k}^{n-(j-1)}\right] B\left(u(t), u_{j}-u(t)\right) k .
\end{aligned}
$$

Each term of the first line of (3.27) is treated in a similar manner. For example,

Now,

$$
\left\|A^{1 / 2} E_{k} B\left(u_{0}, u_{1}\right)\right\| k \leq \frac{C e^{-\delta t}}{t^{1 / 2}}\left\|B\left(u_{0}, u_{1}\right)\right\| k \leq \frac{C\left(\left\|A u_{0}\right\|, \Omega\right) e^{-\delta t}}{t^{1 / 2}} k
$$

$$
\begin{aligned}
& \sum_{j=2}^{n}\left\|A^{1 / 2}\left[e^{-(t-(j-1) k) A}-E_{k}^{n-(j-1)}\right] B\left(u_{j-1}-u(t), u_{j}\right)\right\| k \\
& \quad \leq \sum_{j=2}^{n} \frac{C e^{-\delta(t-(j-1) k)} k}{(t-(j-1) k)^{3 / 2}}\left\|B\left(u_{j-1}-u(t), u_{j}\right)\right\| k \\
& \quad \leq \sum_{j=2}^{n} \frac{C e^{-\delta(t-(j-1) k)} k}{(t-(j-1) k)^{3 / 2}}\left\|A^{1 / 2}\left(u_{j-1}-u(t)\right)\right\|\left\|A u_{j}\right\| k \\
& \quad \leq \sum_{j=2}^{n} \frac{C e^{-\delta(t-(j-1) k)} k}{(t-(j-1) k)^{3 / 2}} \cdot \frac{(t-(j-1) k)}{((j-1) k)^{1 / 2}} e^{-\delta j k} k \\
& \quad=C e^{-\delta t} k\left(\sum_{j=2}^{n} \frac{1}{(t-(j-1) k)^{1 / 2}((j-1) k)^{1 / 2}} k\right) \leq C e^{-\delta t} k
\end{aligned}
$$

again by [18], [25] and Lemma 2 .

The last line of (3.27) is handled similarly and (3.18) is established for $I_{3}$ as well. As anticipated earlier, we thus conclude the proof of the theorem since $\left\|A^{1 / 2}\left(u(t)-u_{n, k}\right)\right\|$ is equivalent to $\left\|u(t)-u_{n, k}\right\|_{1}$, as in [7].

4. Concluding Remarks. Our results are incomplete, just as those of [18], in that the rate of convergence has not been established for $u_{0}$, which is merely assumed to be in $H$, even though the solution exists for any $u_{0} \in H$ [13] since $\Omega \subset \mathbf{R}^{\mathbf{2}}$. It is of interest to consider this more general situation. From the practical standpoint it is perhaps of greater interest to establish higher-order convergence, at least for $u_{0} \in D(A)$, as we mentioned at the beginning.

Department of Mathematical Sciences

San Diego State University

San Diego, California 92182

1. H. AmANN, "Existence and stability of solutions for semilinear parabolic systems and applications to some diffusion reaction equations," Proc. Roy. Soc. Edinburgh Sect. A, v. 81, 1978, pp. 35-47.

2. G. A. BAKER, V. A. Dougalis \& O. A. KARAKAShiAn, "On a higher order accurate fully discrete Galerkin approximation to the Navier-Stokes equations," Math. Comp., v. 39, 1982, pp. 339-375. 
3. M. Crouzeix \& V. Thomée, On the Discretization in Time of Semilinear Parabolic Equations with Non-Smooth Initial Data, Preprint, Université de Rennes, 1985.

4. C. FOIAS \& R. TEMAM, "Some analytic and geometric properties of the solutions of the evolution Navier-Stokes equations," J. Math. Pures Appl., v. 58, 1979, pp. 339-368.

5. H. FUJitA \& T. KATO, "On the Navier-Stokes initial value problem. I," Arch. Rational Mech. Anal., v. 16, 1964, pp. 269-315.

6. H. Fujita \& A. MizUtani, "On the finite element method for parabolic equations, I: Approximation of holomorphic semi-groups," J. Math. Soc. Japan, v. 28, 1976, pp. 749-771.

7. H. Fujita \& H. MoRimoto, "On fractional powers of the Stokes operator," Proc. Japan Acad., v. 46, 1970, pp. 1141-1143.

8. V. Girault \& P. A. RaViart, Finite Element Approximation of Navier-Stokes Equations, Lecture Notes in Math., vol. 749, Springer-Verlag, Berlin and New York, 1979.

9. J. G. HEYWOOD \& R. RANNACHER, "Finite element approximation of the nonstationary Navier-Stokes problem. I. Regularity of solutions and second-order estimates for spatial discretization," SIAM J. Numer. Anal., v. 19, 1982, pp. 275-311.

10. J. G. HEYWOOD \& R. RANNACHER, "Finite element approximation of the nonstationary Navier-Stokes problem, Part II: Stability of solutions and error estimates uniform in time," SIAM J. Numer. Anal., v. 23, 1986, pp. 750-777.

11. J. G. HEYWOOD \& R. RANNACHER, "Finite element approximation of the nonstationary Navier-Stokes problem, Part III. Smoothing property and higher order error estimates for spatial discretization," SIAM J. Numer. Anal., v. 25, 1988, pp. 489-512.

12. J. G. HEYWOOD \& R. RANNACHER, "Finite element approximation of the nonstationary Navier-Stokes problem. IV. Error analysis for second order time discretization," Preprint.

13. J. W. Jerome, Approximation of Nonlinear Evolution Systems, Academic Press, New York and London, 1983.

14. T. KATO, Perturbation Theory for Linear Operators, 2nd ed., Springer-Verlag, Berlin and New York, 1976.

15. T. Kato \& H. Fujita, "On the non-stationary Navier-Stokes system," Rend. Sem. Mat. Univ. Padova, v. 32, 1962, pp. 243-260.

16. M. N. LE ROUX, "Méthodes multipas pour des équations paraboliques non linéaires," Numer. Math., v. 35, 1980, pp. 143-162.

17. H. OKАмото, "On the semi-discrete finite element approximation for the nonstationary Stokes equation," J. Fac. Sci. Univ. Tokyo Sect. IA, v. 29, 1982, pp. 241-260.

18. Н. ОКАмотО, "On the semi-discrete finite element approximation for the nonstationary Navier-Stokes equation," J. Fac. Sci. Univ. Tokyo Sect. IA, v. 29, 1982, pp. 613-651.

19. R. RANNACHER, "Stable finite element solutions to nonlinear parabolic problems of NavierStokes type," in Computing Methods in Applied Sciences and Engineering V (R. Glowinski and J. L. Lions, eds.), North-Holland, Amsterdam, 1982, pp. 301-309.

20. R. RAUTMANN, "A semigroup approach to error estimates for nonstationary Navier-Stokes approximations," Methoden Verfahren Math. Phys., v. 27, 1983, pp. 63-77.

21. R. RAUTMANN, "On optimum regularity of Navier-Stokes solutions at time $t=0$," Math. $Z$., v. 184,1983 , pp. 141-149.

22. R. TEMAM, Navier-Stokes Equations, Theory and Numerical Analysis, 2nd ed., North-Holland, Amsterdam, 1979.

23. R. TEMAM, "Behaviour at time $t=0$ of the solutions of semilinear evolution equations," $J$. Differential Equations, v. 43, 1982, pp. 73-92.

24. R. TEMAM, Navier-Stokes Equations and Nonlinear Functional Analysis, CBMS-NSF Regional Conf. Ser. in Applied Math., vol. 41, SIAM, Philadelphia, 1983.

25. V. THOMÉ, Galerkin Finite Element Methods for Parabolic Problems, Lecture Notes in Math., vol. 1054, Springer-Verlag, Berlin and New York, 1984. 